\title{
Exploring the physics of core-collapse supernovae with multidimensional simulations: from axisymmetry to three dimensions
}

\author{
Alexander Summa ${ }^{1}$, Hans-Thomas Janka ${ }^{1}$, Florian Hanke ${ }^{1,2}$, \\ Tobias Melson ${ }^{1,2}$, Andreas Marek ${ }^{3}$ and Bernhard Müller ${ }^{4,5}$ \\ ${ }^{1}$ Max-Planck-Institut für Astrophysik, \\ Karl-Schwarzschild-Str. 1, 85748 Garching, Germany \\ email: asumma@mpa-garching.mpg.de \\ ${ }^{2}$ Physik Department, Technische Universität München, \\ James-Franck-Straße 1, 85748 Garching, Germany \\ ${ }^{3}$ Max Planck Computing and Data Facility (MPCDF), \\ Gießenbachstr. 2, 85748 Garching, Germany \\ ${ }^{4}$ Astrophysics Research Centre, School of Mathematics and Physics, \\ Queen's University Belfast, Belfast, BT7 1NN, United Kingdom \\ ${ }^{5}$ Monash Centre for Astrophysics, School of Physics and Astronomy, \\ Monash University, Victoria 3800, Australia
}

\begin{abstract}
Multidimensional effects are essential for the success of the neutrino-driven explosion mechanism of core-collapse supernovae. Although astrophysical phenomena in nature involve three spatial dimensions, the huge computational demands still allow only for a few self-consistent, three-dimensional (3D) simulations focusing on specific aspects of the explosion physics, whereas systematic studies of larger sets of progenitor models or detailed investigations of different explosion parameters are restricted to the axisymmetric (2D) modeling approach at the moment. Employing state-of-the-art neutrino physics, we present the results of self-consistent core-collapse supernova simulations performed with the Prometheus-Vertex code in 2D and 3D. The $2 \mathrm{D}$ study of 18 successfully exploding pre-supernova models in the range of 11 to 28 solar masses shows the progenitor dependence of the explosion dynamics: if the progenitor exhibits a pronounced decline of the density at the $\mathrm{Si} / \mathrm{Si}-\mathrm{O}$ composition shell interface, the rapid drop of the mass-accretion rate at the time the interface arrives at the shock induces a steep reduction of the accretion ram pressure. This causes a strong shock expansion supported by neutrino heating and thus favors an early explosion. In case of a more gradually decreasing accretion rate, it takes longer for the neutrino heating to overcome the accretion ram pressure and explosions set in later. By considering the effects of turbulent pressure in the gain layer, we derive a generalized condition for the critical neutrino luminosity that captures the explosion behavior of all models very well. We show that this concept can also be extended to describe the effects of rotation as well as the behavior of recent 3D simulations and that the conditions necessary for the onset of explosion can be defined in a similar way.
\end{abstract}

Keywords. supernovae: general, hydrodynamics, turbulence, neutrinos 